\title{
Factores de riesgo de los trastornos de la conducta alimentaria en jóvenes escolarizados en Cundinamarca (Colombia)*
}

\author{
Sandra Piñeros Ortiz ${ }^{1}$ \\ Jenny Molano Caro ${ }^{2}$ \\ Clara López de Mesa ${ }^{3}$
}

\begin{abstract}
Resumen
Introducción: El diagnóstico precoz de un trastorno de la conducta alimentaria (TCA) es determinante para evitar complicaciones graves a lo largo de la vida. Pocos estudios en Colombia han examinado sus características clínicas y comorbilidad en la población escolar. Objetivo: Determinar la frecuencia de TCA, sus factores de inicio y mantenimiento, así como sus subtipos clínicos y comorbilidad en una población escolarizada. Método: Estudio de base comunitaria y corte transversal con 937 estudiantes entre 12 y 20 años de edad, de Bogotá y la sabana centro de Cundinamarca, que respondieron la EAT-26, encuesta de factores de inicio y mantenimiento de TCA, y la Zung de ansiedad y depresión. Los casos probables fueron contactados para entrevista clínica psiquiátrica. Resultados: Se detectaron 141 casos probables de TCA, la mayoría en mujeres ( $\mathrm{p}<0,01 \equiv 38,3 \%$ de ellos aceptó entrevista psiquiátrica. Se realizó diagnóstico clínico de TCA en el 53,7\% de los entrevistados, sin diferencias por estrato socioeconómico ni grupos de edad. El subtipo no especificado estuvo presente en el 93\%. Se encontró comorbilidad con trastornos depresivos y ansiosos, así como conductas suicidas más frecuentes en adolescentes con diagnóstico clínico de TCA que en aquellos sin diagnóstico psiquiátrico y con otros trastornos $(\mathrm{p}=0,019)$. Conclusión: Los TCA ocurren desde edades tempranas. Existe una alta tasa de comorbilidad con trastornos afectivos y conductas suicidas.
\end{abstract}

Palabras clave: trastornos de la conducta alimentaria, adolescente, comorbilidad, factores epidemiológicos.

* El estudio fue aprobado por la subcomisión de Investigación de la Facultad de Medicina de la Universidad de La Sabana y costeado por el Fondo Patrimonial para la Investigación, de la Universidad de La Sabana.

1 Médica psiquiatra. Especialista en Psiquiatría infantil y de Adolescentes. Profesora clínica, Facultad de Medicina Universidad de La Sabana. Docente ocasional del Departamento Psiquiatría de la Universidad Nacional de Colombia. Bogotá, Colombia.

2 Médica internista, gastroenteróloga. Profesora de la Facultad de Medicina Universidad de La Sabana. Bogotá, Colombia.

3 Estadística. Profesora Facultad de Medicina Universidad de La Sabana. Bogotá, Colombia. 
Title: Risk Factors for Eating Disorders among School-Aged Young People in Cundinamarca (Colombia)

\section{Abstract}

Introduction: The early diagnosis of Eating Disorders (ED) is decisive in order to prevent serious complications during lifetime. In Colombia, few studies have assessed the clinical characteristics and comorbidity of ED in students. Objective: To determine the frecuency of ED, its onset and maintenance factors, as well as its clinical subtypes and comorbidity in student population. Method: Cross sectional and community based study with 937 students, ages 10 to 12, from Bogotá and Sabana Centro-Cundinamarca, who answered the EAT- 26, a survey on onset and maintenance factors for ED, and the Zung depression and anxiety scales. Results: 141 probable cases of ED were detected, predominantly among women ( $\mathrm{p}<0.01) .38 .3 \%$ of them accepted clinical psychiatric evaluation. ED was clinically diagnosed in $53.7 \%$ of the cases, without significant differences regarding socioeconomic level or age groups; subtype not otherwise specified in $93 \%$. Comorbidity with depression and anxiety disorders was also found; similarly, suicidal behaviors were more frequent in adolescents with clinical diagnosis of ED than in those without psychiatric diagnosis or in those with other psychiatric disorders ( $p=0.019)$. Conclusions: ED occurs since early ages. Comorbidity with affective disorders and suicidality is high.

Key words: Eating disorders, adolescent, comorbidity, epidemiologic factors.

\section{Introducción}

La aparición de los trastornos de la conducta alimentaria (TCA) y de sus conductas precursoras a edades tempranas ha despertado gran preocupación durante las últimas décadas en diversos sectores sociales de países occidentales y no occidentales (1). Profesionales de la salud, de la educación y de los medios de comunicación han hecho grandes esfuerzos por adelantar acciones de promoción, prevención primaria y secundaria a ese respecto, con el fin de abordar a la población más vulnerable y no diagnosticada $(2,3)$.

A pesar de las limitaciones de los programas preventivos, la importancia del diagnóstico temprano no deja de destacarse, toda vez que los estudios sobre pronóstico muestran resultados más favorables en adolescentes que en adultos, en términos de recuperación de los síntomas nucleares y de mortalidad (4).

Los TCA no especificados (NOS), atípicos o formas parciales son los más habituales en la comunidad $y$ en centros de atención primaria, con datos de prevalencia del 4\% al $22 \%$. Esta categoría agrupa los casos que no reúnen los criterios diagnósticos estrictos para anorexia nerviosa (AN) y bulimia nerviosa (BN), pero sus manifestaciones son de la relevancia clínica suficiente para requerir intervención y seguimiento. Algunos de ellos evolucionan a AN o BN clásicas (5).

Más de la mitad de los pacientes con AN tienen conductas bulimicas, y a lo largo del tiempo pueden recuperar peso. La historia natural indica que no existe una estabilidad diagnóstica entre las distintas categorias planteadas. Así mismo, el patrón de comorbilidad psiquiátrica es similar en las tres entidades, lo cual ha pues- 
to aún más en duda su naturaleza categórica y ha llevado a plantear aproximaciones dimensionales, que abordan este problema como un continuo (6). Esta hipótesis resulta atractiva y útil en la niñez tardía y la adolescencia, etapa durante la cual son usuales comportamientos de riesgo como hacer dietas y otras prácticas inapropiadas para controlar el peso y la figura. Si bien en la mayoría de los casos son normativas, estas prácticas podrian corresponder a manifestaciones tempranas, factores de inicio y mantenimiento de TCA. De ahí la importancia de interpretar con cautela resultados de estudios basados exclusivamente en la aplicación de cuestionarios de tamizaje o en hallazgos aislados de alteraciones en la alimentación, la imagen y el peso corporal.

En Colombia se ha encontrado un alto porcentaje de síntomas de TCA en estudiantes de secundaria de Medellin (7). Se ha investigado la prevalencia de TCA en población universitaria de Bogotá y en jóvenes escolarizadas de Bucaramanga utilizando instrumentos como el ECA y el SCOFF $(8,9)$. El ECA es una encuesta relativamente extensa, de 32 preguntas, diseñada por los autores del estudio y aplicada en estudiantes de la Universidad Nacional de Colombia, y que, por la complejidad de algunas de sus preguntas, puede ser de difícil comprensión para estudiantes de secundaria. E1 SCOFF fue validado recientemente en Bucaramanga, Colombia, $y$ aunque hasta el momento no se ha aplicado en otras regiones del país, ofrece la ventaja de ser más breve y sencillo (10).

La prevalencia de TCA en estudiantes de secundaria de Bucaramanga y en jóvenes de la Universidad Nacional de Bogotá fue del 30\%, distribuida de la siguiente forma: AN: $1,7 \%$ y $0,48 \%$; BN: $6 \%$ y $1,2 \%$; y TCA NOS: $22,3 \%$ y $28 \%$, respectivamente $(8,9)$.

Los síntomas más frecuentes, el patrón de comorbilidad, los factores precipitantes y de mantenimiento de TCA se han examinado ampliamente en países industrializados, tanto en estudios de base comunitaria como en población clínica (11-16).

En Colombia tales estudios se han basado, fundamentalmente, en población clínica, de centros de atención terciaria o especializada, constituida en su mayoría por adultos jóvenes. $(17,18)$.

Las personas con TCA tienen una alta prevalencia de conductas suicidas. El riesgo de suicidio en mujeres con AN es aproximadamente 20 veces mayor que el de la población general, y en pacientes con BN entre el $15 \%$ y el $40 \%$ ha incurrido en por lo menos un intento de suicidio $(19,20)$. Existen limitaciones metodológicas que no permiten extrapolar los resultados de estos estudios a la población escolar y adolescente de Colombia. Por ejemplo, el estudio en adolescentes escolarizadas de Medellín examinó síntomas de estos trastornos sólo en mujeres (7). 
El promedio de edad de la población estudiada en La Universidad Nacional de Colombia $(21,6 \pm 0,21$

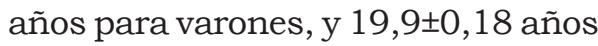
para mujeres) sobrepasó la franja de edad de mayor frecuencia de estas entidades (8). Estudios epidemiológicos han encontrado un descenso de la incidencia a niveles prepuberales a partir de los 18 años para AN, y a partir de los 20 años para BN (11).

Es importante examinar la frecuencia de estos trastornos y sus conductas precursoras en mujeres y en hombres; especialmente, en un grupo de edad considerado de alto riesgo, y sobre el cual se puedan aplicar estrategias preventivas tanto primarias como secundarias. Así mismo, es importante examinar las características clínicas, los factores relacionados con el inicio y mantenimiento y las conductas suicidas en muestras de la comunidad, pues en la literatura colombiana esta información proviene de muestras clinicas, en las que se supone mayor tiempo de evolución de la enfermedad, así como mayor compromiso clínico y funcional (20).

A partir de estos hallazgos surge la necesidad de abarcar una población más joven y estratificada. Esto, con el objetivo de detectar precozmente a los sujetos en riesgo y afectados, y establecer el diagnóstico diferencial y los factores asociados a la aparición y el mantenimiento de dichos trastornos. Lo anterior puede contribuir a que mejore la calidad de vida de la población vulnerable y aliviar los costos sociales derivados de las complicaciones inherentes a esta problemática; posiblemente, a través de acciones de divulgación y educación a los jóvenes, a sus familias y a la comunidad escolar y científica, así como a las instituciones gubernamentales.

El objetivo del presente estudio fue determinar la frecuencia de TCA en jóvenes escolarizados de Bogotá y Sabana Centro de Cundinamarca, los factores asociados a su inicio y mantenimiento, las formas de presentación clínica, la comorbilidad psiquiátrica, la asociación a conductas suicidas y el diagnóstico diferencial.

\section{Métodos}

El presente estudio hace parte de la investigación Epidemiología de trastornos ácido-pépticos y de la conducta alimentaria en jóvenes escolarizados de 12 a 20 años de edad, de Bogotá y la Sabana Centro de Cundinamarca. Los resultados de trastornos ácido-pépticos y los factores emocionales e impacto social y escolar de estos fueron publicados en otra fuente. $(21,22)$. Es un estudio de base comunitaria y de corte transversal. Se realizó en la ciudad de Bogotá y el área de la Sabana Centro del Departamento de Cundinamarca, en los municipios de Chía, Tabio, Cajicá y Sopó, en un área geográfica andina rural y urbana. La recolección de los datos se llevó a cabo durante el segundo semestre de 2005. 
Se seleccionó aleatoriamente a 35 instituciones educativas de la zona geográfica mencionada; de ellas, 12 aceptaron participar. Se excluyeron las instituciones para población con necesidades educativas especiales.

La muestra se calculó mediante el programa estadístico Tamu, con una frecuencia esperada de TCA del $22 \%$, error alfa del $0,05 \%$, un poder del $90 \%$ y un porcentaje alrededor de la muestra del 2,4\%. El número de sujetos fue 857 . Se solicitaron los listados de los jóvenes de 12 a 20 años matriculados y se seleccionó aleatoriamente a 1.100 , de los cuales 937 aceptaron participar. Se obtuvo consentimiento informado escrito en los jóvenes mayores de 18 años y en los padres o tutores de los menores de 18 años, con previa información sobre la naturaleza de los procedimientos, los riesgos y los beneficios de la investigación, y sin coacción de ningún tipo. Se obtuvo también asentimiento informado en los menores de 18 años.

Los sujetos participantes respondieron los siguientes instrumentos:

- Encuesta autodiligenciada de factores asociados al desarrollo y mantenimiento de TCA, identificados en la literatura mundial (13). La encuesta la conforma un total de 21 items; 17 de ellos, con opciones de respuesta "Sí", "No" y "No sé", corresponden a la percepción del peso y la figura propias, vegetarianismo, percepción de críticas familiares acerca del peso corporal y comer en secreto; también, antecedentes familiares de AN, BN, obesidad, insatisfacción con el peso, insatisfacción con la figura, realización de dietas sin prescripción médica y pérdida de control sobre la ingesta de comida. Hay también dos ítems sobre conocimientos de alimentación saludable; específicamente, sobre grasas y carbohidratos, con opciones de respuesta "Si" y "No", y dos items adicionales sobre la relación delgadez-éxito, delgadez-aprobación externa, para responder según una escala likert (de "Totalmente de acuerdo" a "Totalmente en desacuerdo").

- Cuestionario EAT-26. Instrumento de autoinforme ampliamente utilizado y validado en diferentes culturas, que permite detectar casos probables de AN, BN y TCA NOS. Es una versión abreviada del EAT-40, elaborada por Garner y Garfinkel, con un punto de corte de 20 $(23,24)$. La sensibilidad y la especificidad varian de acuerdo con el contexto cultural de validación, pero, en general, son altas: por ejemplo, en España, para un punto de corte de 20 la sensibilidad es del $91 \%$, y la especificidad, del 69\%. Se recomienda su uso en investigacio- 
nes de dos fases, en las cuales los individuos con puntajes iguales o superiores a 20 sean luego evaluados con una entrevista diagnóstica. Una versión del EAT-26 que se encontraba en proceso de validación en Colombia durante la realización de esta investigación fue utilizada en este estudio. Posteriormente, en 2007, se publicó el EAT- 26-M (escala abreviada y modificada de las actitudes alimentarias), instrumento validado en Colombia en población de 9 a 17 años de edad, con una confiabilidad, medida a través del alfa de crohnbach, de 0,87 , IC $95 \%(0,86-0,88)(25)$.

- Cuestionarios de autoinforme de Zung para ansiedad y depresión. Adaptados y utilizados en población colombiana. Su confiabilidad es mayor en adultos jóvenes (26-28).

Los jóvenes con un resultado del cuestionario EAT- $26 \geq 20$, (casos probables de TCA) fueron contactados telefónicamente para valoración con entrevista clínica estructurada basada en los criterios del DSMIV-R, con el fin de confirmar o descartar diagnóstico de AN, BN, TCA NOS, establecer la comorbilidad o el diagnóstico diferencial con otros trastornos psiquiátricos, $\mathrm{y}$ conductas suicidas.

La información fue almacenada en una base de datos de Excel, previamente validada para procesar los datos en el programa estadístico SPSS versión 14.0. Los resultados se presentan en tablas para variables de escala de medición nominal y ordinal. Se aplicaron pruebas estadísticas de asociación como $\chi^{2}$ para proporciones, y Mann- Whitney para distribuciones no paramétricas; se calcularon OR e intervalos de confianza del 95\%.

\section{Resultados}

Participaron 937 jóvenes: el $40,4 \%$ eran hombres, y el $59,6 \%$, mujeres. El 44,1\% eran de instituciones educativas de Bogotá, y el $55,9 \%$, de municipios de La Sabana Centro de Cundinamarca. El 49\% eran de los estratos socioeconómicos 1 a 3 , y el $50,6 \%$, de los estratos más altos.

Primera fase, o fase de tamizaje

El 15,1\% ( $n=141)$ de los jóvenes tuvo diagnóstico probable de TCA mediante la aplicación del instrumento EAT- 26, con puntaje $\geq 20$. El $84,4 \%$ de los casos probables fueron mujeres, con una diferencia significativa respecto a los hombres $(p=0,01)$. El $59,6 \%$ procedia de algún estrato alto $(p=0,02)$. No se encontró diferencia estadísticamente significativa en la frecuencia de diagnóstico probable de TCA por localización de la institución educativa ni por edad (Tabla 1). 
Tabla 1. Distribución porcentual de jóvenes según cuestionario EAT y variables sociodemográficas

\begin{tabular}{|c|c|c|c|c|c|c|c|c|}
\hline & \multicolumn{4}{|c|}{ Cuestionario EAT Puntaje } & \multirow{3}{*}{$\mathbf{p}$} & \multirow{3}{*}{ OR } & \multirow{3}{*}{ IC(95\%) } \\
\hline & & \multicolumn{2}{|c|}{$<20$} & \multicolumn{2}{|c|}{$\geq 20$} & & & \\
\hline & & $\mathbf{n}$ & $\%$ & $\mathbf{n}$ & $\%$ & & & \\
\hline \multirow{3}{*}{ Género } & Femenino & 437 & 55,1 & 119 & 84,4 & \multirow{2}{*}{0,001} & \multirow{2}{*}{4,41} & \multirow{2}{*}{$2,68-7,31$} \\
\hline & Masculino & 356 & 44,9 & 22 & 15,6 & & & \\
\hline & Total & 793 & 100 & 141 & 100 & & & \\
\hline \multirow{3}{*}{ Estrato } & Bajo & 404 & 51,2 & 57 & 40,4 & \multirow{2}{*}{0,01} & \multirow{2}{*}{1,55} & \multirow{2}{*}{$1,06-2,26$} \\
\hline & Alto & 385 & 48,8 & 84 & 59,6 & & & \\
\hline & Total & 789 & 100 & 141 & 100 & & & \\
\hline
\end{tabular}

La proporción de jóvenes con sintomas depresivos y ansiosos de relevancia clínica según el cuestionario de Zung fue del 40,3\% y del $35,4 \%$, respectivamente. El diagnóstico probable de ansiedad y depresión, evaluado mediante este cuestionario, fue mayor en los jóvenes con un EAT- 26 20 : el 68,1\% para depresión, y el 67,4\% para ansiedad $(p=0,001)$ (Tabla 2).
Para el análisis de los factores de riesgo de desarrollo y mantenimiento de TCA descritos en la literatura y seleccionados en la encuesta aplicada en esta fase, se excluyeron las respuestas "No sé" y sólo se procesaron "Sí" y "No", por lo cual los valores totales de respuestas a cada pregunta son diferentes (tablas 3 y 4).

Tabla 2. Distribución porcentual de jóvenes escolarizados según diagnóstico probable de TCA y presencia de sindromes ansioso y depresivo

\begin{tabular}{|c|c|c|c|c|c|c|c|}
\hline & \multicolumn{4}{|c|}{ Cuestionario EAT Puntaje } & \multirow{3}{*}{$\mathbf{P}$} & \multirow{3}{*}{ OR } & \multirow{3}{*}{ IC(95\%) } \\
\hline & \multicolumn{2}{|c|}{$<20$} & \multicolumn{2}{|c|}{$\geq 20$} & & & \\
\hline & $\mathbf{n}$ & $\%$ & $\mathbf{n}$ & $\%$ & & & \\
\hline Total & 793 & 100 & 141 & 100 & & & \\
\hline Depresión & 280 & 35,3 & 96 & 68,1 & 0,001 & 3,91 & $2,62-5,84$ \\
\hline Ansiedad & 236 & 29,8 & 95 & 67,4 & 0,001 & 4,87 & $3,27-7,29$ \\
\hline
\end{tabular}


Tabla 3. Distribución porcentual de jóvenes según cuestionario EAT y presencia factores familiares relacionados con inicio y mantenimiento de TCA

\begin{tabular}{|c|c|c|c|c|c|c|c|}
\hline \multirow{2}{*}{ Factores familiares } & \multicolumn{2}{|c|}{ EAT $<20$} & \multicolumn{2}{|c|}{ EAT $\geq 20$} & \multirow{2}{*}{$\mathbf{p}$} & \multirow{2}{*}{ OR } & \multirow{2}{*}{ IC(95\%) } \\
\hline & $\mathbf{n}$ & $\%$ & $\mathbf{n}$ & $\%$ & & & \\
\hline Antecedentes de anorexia & 42 & 6,9 & 15 & 14,7 & 0,007 & 2,34 & $1,19-4,58$ \\
\hline Bulimia & 33 & 5,5 & 16 & 16,7 & 0,001 & 3,41 & $1,71-6,76$ \\
\hline $\begin{array}{l}\text { Críticas y bromas familiares } \\
\text { sobre la figura }\end{array}$ & 196 & 27,5 & 70 & 53,8 & 0,001 & 3,08 & $2,07-4,60$ \\
\hline $\begin{array}{l}\text { Críticas y bromas familiares } \\
\text { sobre el peso }\end{array}$ & 186 & 25,2 & 64 & 50 & 0,001 & 2,97 & $1,99-4,45$ \\
\hline $\begin{array}{l}\text { Insatisfacción con la figura } \\
\text { en familiares }\end{array}$ & 287 & 44,3 & 84 & 72,4 & 0,001 & 3,3 & $2,09-5,23$ \\
\hline $\begin{array}{l}\text { Insatisfacción con el peso en } \\
\text { familiares }\end{array}$ & 303 & 47,4 & 91 & 73,4 & 0,001 & 3,06 & $1,96-4,80$ \\
\hline $\begin{array}{l}\text { Antecedentes familiares de } \\
\text { obesidad }\end{array}$ & 353 & 48,6 & 74 & 59,7 & 0,02 & 1,56 & $1,04-2,253$ \\
\hline $\begin{array}{l}\text { Antecedentes familiares de } \\
\text { dietas }\end{array}$ & 409 & 58,9 & 104 & 81,9 & 0,001 & 3,15 & $1,91-5,22$ \\
\hline $\begin{array}{l}\text { Antecedentes familiares de } \\
\text { pérdida de control de inges- } \\
\text { ta alimentaria }\end{array}$ & 234 & 34,6 & 65 & 54,2 & 0,001 & 2,24 & $1,48-3,38$ \\
\hline Total & 677 & 100 & 120 & 100 & & & \\
\hline
\end{tabular}

Tabla 4. Distribución porcentual de jóvenes según cuestionario EAT y presencia factores individuales relacionados con inicio y mantenimiento de TCA

\begin{tabular}{|c|c|c|c|c|c|c|c|}
\hline \multirow{2}{*}{ Factores individuales } & \multicolumn{2}{|c|}{ EAT $t \geq 20$} & \multicolumn{2}{|c|}{ EAT $<20$} & \multirow{2}{*}{$\mathbf{p}$} & \multirow{2}{*}{ OR } & \multirow{2}{*}{ IC(95\%) } \\
\hline & $\mathbf{n}$ & $\%$ & $\mathbf{n}$ & $\%$ & & & \\
\hline Percepción de sobrepeso & 78 & 60 & 138 & 18,6 & 0,001 & 6,54 & $4,32-9,92$ \\
\hline $\begin{array}{l}\text { Percepción de rechazo por la fi- } \\
\text { gura }\end{array}$ & 24 & 19,8 & 36 & 4,9 & 0,001 & 4,81 & $2,65-8,71$ \\
\hline $\begin{array}{l}\text { Insatisfacción global con la figura } \\
\text { corporal }\end{array}$ & 31 & 25 & 56 & 7,4 & 0,001 & 4,15 & $2,47-6,95$ \\
\hline $\begin{array}{l}\text { Insatisfacción global con el tamaño } \\
\text { corporal }\end{array}$ & 44 & 34,9 & 61 & 8,1 & 0,001 & 6,11 & $3,80-9,83$ \\
\hline $\begin{array}{l}\text { Insatisfacción parcial con la figura } \\
\text { corporal }\end{array}$ & 91 & 70 & 270 & 35,6 & 0,001 & 4,22 & $2,77-6,44$ \\
\hline $\begin{array}{l}\text { Insatisfacción parcial con el tamaño } \\
\text { corporal }\end{array}$ & 86 & 66,2 & 202 & 26,6 & 0,001 & 5,39 & $3,56-8,18$ \\
\hline Vegetarianismo & 10 & 7,8 & 43 & 5,6 & & & \\
\hline $\begin{array}{l}\text { Ingestión alimentaria excesiva en } \\
\text { secreto }\end{array}$ & 35 & 26,9 & 99 & 13 & 0,001 & 2,46 & $1,55-3,92$ \\
\hline Total & 130 & 100 & 761 & 100 & & & \\
\hline
\end{tabular}


En los jóvenes con EAT- 26 $\geq 20$ (probable TCA) hubo una relación significativa con los siguientes factores de riesgo: antecedentes familiares de AN, BN, obesidad y realización de dietas; insatisfacción personal y familiar con figura y peso corporal, y críticas y comentarios familiares negativos sobre el peso y la figura. $(p<0,05)$ (tablas 3 y 4$)$.

El grupo con probable TCA tuvo más respuestas incorrectas acerca de la importancia de las grasas y carbohidratos en una alimentación saludable. Un 43,6\% de ellos manifestó estar de acuerdo y totalmente de acuerdo con la afirmación "Hay que ser delgado para tener éxito en la vida", frente a un $10,8 \%$ de los jóvenes con EAT- $26<20$, con una diferencia estadísticamente significativa $(p=0,001)$. Respecto a la afirmación "Hay que ser delgado para agradar a los demás", un 37,6\% de los jóvenes con probable TCA manifestó estar de acuerdo y totalmente de acuerdo con ella, frente a un $11,1 \%$ de los jóvenes con EAT-26<20 (Tabla 5).

Tabla 5. Distribución porcentual de jóvenes según cuestionario EAT y conocimientos $y$ actitudes relacionados con figura corporal

\begin{tabular}{|c|c|c|c|c|c|c|}
\hline & \multicolumn{2}{|l|}{$<20$} & \multicolumn{2}{|c|}{$\geq 20$} & \multirow{2}{*}{$\mathbf{p}$} & \multirow[b]{2}{*}{0,028} \\
\hline & $\mathbf{n}$ & $\%$ & $\mathbf{n}$ & $\%$ & & \\
\hline \multicolumn{2}{|c|}{$\begin{array}{l}\text { Conocimiento sobre el papel de los carbohidratos en } \\
\text { una alimentación saludable }\end{array}$} & 457 & 72,2 & 74 & 62,2 & 0,028 \\
\hline \multicolumn{2}{|c|}{$\begin{array}{l}\text { Conocimiento sobre el papel de las grasas en una } \\
\text { alimentación saludable }\end{array}$} & 253 & 38,8 & 34 & 28,8 & 0,039 \\
\hline \multirow{6}{*}{$\begin{array}{l}\text { Actitud hacia la del- } \\
\text { gadez relacionada } \\
\text { con el éxito }\end{array}$} & Totalmente de acuerdo & 27 & 3,5 & 17 & 12,8 & \multirow{5}{*}{0,001} \\
\hline & De acuerdo & 57 & 7,3 & 41 & 30,8 & \\
\hline & Indiferente & 222 & 28,6 & 31 & 23,3 & \\
\hline & En desacuerdo & 214 & 27,6 & 26 & 19,5 & \\
\hline & Totalmente en desacuerdo & 256 & 33,0 & 18 & 13,5 & \\
\hline & Total & 776 & 100,0 & 133 & 100,0 & \\
\hline \multirow{6}{*}{$\begin{array}{l}\text { Actitud hacia la del- } \\
\text { gadez relacionada } \\
\text { con el agrado a los } \\
\text { demás }\end{array}$} & Totalmente de acuerdo & 20 & 2,6 & 15 & 11,3 & \\
\hline & De acuerdo & 65 & 8,5 & 35 & 26,3 & \\
\hline & Indiferente & 186 & 24,2 & 31 & 23,3 & \\
\hline & En desacuerdo & 215 & 28,0 & 31 & 23,3 & \\
\hline & Totalmente en desacuerdo & 282 & 36,7 & 21 & 15,8 & \\
\hline & Total & 768 & 100,0 & 133 & 100,0 & \\
\hline
\end{tabular}


Segunda fase, o fase de entrevista clinica estructurada

De los 141 casos probables de TCA detectados con el EAT-26, un $38,3 \%(54 / 141)$ aceptó la realización de la entrevista clínica estructurada psiquiátrica. Con base en los resultados de esta entrevista se diagnosticó TCA en el 53,7\% (29/54) de los casos, y otros diagnósticos psiquiátricos en ausencia de TCA, en el 13\% (7/54); no se encontraron TCA ni otros trastornos psiquiátricos en el 33,3\% (18/54).

El $81,5 \%(n=44)$ de los jóvenes entrevistados fueron mujeres, y de éstas, el 63,6\% $(n=28)$ tuvo TCA, que fue más habitual en mujeres (96\%).

Aunque el diagnóstico de TCA por entrevista clínica se presentó más en el grupo de edad de 15 a 17 años $(44,8 \%)$ y en el estrato socioeconómico bajo $(58,6 \%)$, esas 2 variables no fueron estadísticamente significativas al compararlas con los jóvenes con trastornos psiquiátricos distintos de TCA, ni con aquellos sin ningún diagnóstico psiquiátrico.

Del total de jóvenes con diagnóstico de TCA, el 93,1\% tuvo TCA NOS, y el $6,9 \%$, BN. Uno de los jóvenes con TCA NOS había reunido previamente los criterios diagnósticos de AN, pero al momento de la entrevista ya no los cumplía totalmente.

Los trastornos psiquiátricos comórbidos con TCA más frecuentes fueron: trastornos depresivos: $23,3 \%$ (7/29); trastornos de ansiedad: $10 \%$
(3/29); trastornos adaptativos: 3,3\% $(1 / 29)$. Una percepción negativa de las relaciones familiares también fue más asidua en los jóvenes con diagnóstico de TCA (43,3\%).

Los diagnósticos psiquiátricos distintos de TCA que se encontraron en el $13 \%(7 / 54)$ de los sujetos entrevistados fueron: trastorno dismorfofóbico (2/7), maltrato emocional $(2 / 7)$ y disfunción familiar (3/7).

De los 29 jóvenes con diagnóstico de TCA por entrevista clínica, 14 habian tenido conductas del espectro suicida a lo largo de su vida; 13 de ellos, ideación, planes y amenazas; y uno, intento de suicidio. De los 7 sujetos con otros diagnósticos psiquiátricos sólo uno tuvo ideas, planes e intento suicida, y ninguno de los 18 sujetos sin diagnóstico psiquiátrico tuvo conductas del espectro suicida $(p=0,019)$.

\section{Discusión}

La frecuencia de TCA encontrada en el presente estudio en la fase de tamizaje $(15,1 \%)$ concuerda con la de estudios de otros países, en los cuales se ha utilizado el EAT- 26 para estimar la prevalencia en población general o de cuidados primarios. En un estudio de King y cols. el 13,3\% de mujeres y el 2,7\% de hombres de una muestra de 720 sujetos que respondieron este cuestionario tuvieron puntaje superior a $20(29,30)$.

El EAT- 26 utilizado en nuestro estudio se encuentra en proceso de validación. Es posible que existan 
diferencias en la frecuencia de TCA en investigaciones basadas en población escolar en las que se utilice el EAT-26-M. Esta versión fue validada en estudiantes de secundaria de Colombia y publicada después de la recolección de la muestra y la aplicación de los instrumentos en este trabajo (25). Sin embargo, para conocer estas diferencias sería necesario realizar investigaciones comparativas acerca de las propiedades psicométricas de estas dos versiones.

Las características sociodemográficas y clínicas de TCA en la población estudiada son semejantes a las de investigaciones internacionales y nacionales, con predominio en mujeres y mayor proporción de formas no especificadas sobre BN y AN clásicas $(8,9)$.

Aunque en nuestro estudio más de la mitad de los jóvenes con probable TCA procedian de estratos socioeconómicos altos, la relación con esta variable dejó de ser significativa en los casos diagnosticados con entrevista clínica. Este hallazgo, junto con la distribución homogénea de los casos por localización de la institución educativa, discriminada como urbana o rural, es consistente con datos epidemiológicos, los cuales indican que este problema no es exclusivo de estratos altos, y se ha ido extendiendo a contextos culturales considerados menos susceptibles a las influencias de estereotipos de belleza y delgadez (31).

La correlación entre puntajes del EAT- 26 y los cuestionarios de
Zung coincide con datos del estudio realizado en estudiantes de la Universidad Nacional de Colombia (8).

En una investigación hecha a 10 pacientes pediátricos con TCA atendidos en centros de tercer nivel de Bogotá, la comorbilidad más usual fue trastorno obsesivo compulsivo (TOC) y depresión; se identificó obesidad familiar como un antecedente significativo, y hacer dietas, como un factor precipitante (18).

La asociación entre TCA y trastornos depresivos y ansiosos se confirmó también en este estudio, con la entrevista clínica. Dicha asociación ha sido ampliamente documentada en investigaciones internacionales. Estudios de gemelos han evidenciado una vulnerabilidad genética común entre ansiedad y TCA, y una relación entre trastornos de ansiedad en la infancia, depresión y síntomas de patología alimentaria, que se mantiene a lo largo de todo el desarrollo $(32,33)$.

Los trastornos de ansiedad en la infancia parecen tener una influencia en la expresión de AN; específicamente, en la aparición de conductas purgativas y en la severidad clínica. También se ha encontrado una asociación importante entre TCA, TOC y depresión a lo largo de la vida, por lo cual se ha planteado que tales trastornos en la infancia serian mecanismos psicopatológicos que conducen a la expresión de TCA $(34,35)$. Aunque esta relación no fue examinada en el presente estudio, vale la pena explorarla en futuras 
investigaciones y considerarla en las intervenciones a población infantil

En este estudio no se encontró comorbilidad con trastornos relacionados con uso de sustancias psicoactivas, hallazgo que contrasta con investigaciones en Colombia, como la realizada en pacientes ambulatorios de un programa especializado en el manejo de TCA, durante la cual se detectó abuso o dependencia en el $18,7 \%$ de los casos (20). Esta diferencia se explicaría por la mayor probabilidad de la población que recibe tratamiento en instituciones especializadas de tener comorbilidad con abuso o dependencia a sustancias, así como con otros trastornos psiquiátricos.

El trastorno dismorfofóbico, diagnosticado en dos sujetos con EAT$26>20$, es uno de los diagnósticos diferenciales de TCA para tener en cuenta, de acuerdo con los resultados del presente estudio. Ambos comparten características esenciales, y en ocasiones su diferenciación es compleja. Otros diagnósticos diferenciales fueron maltrato emocional y disfunción familiar, hechos en dos y tres sujetos, respectivamente, sin diagnóstico clínico de TCA. Una explicación tentativa de este hallazgo tendría que ver con la influencia de los aspectos relacionales y de los estresores psicosociales en el desarrollo de la imagen corporal y la autoestima, así como en su impacto directo sobre la conducta alimentaria.

Resultados del estudio realizado en pacientes ambulatorios de un programa especializado en el manejo de TCA de Bogotá mostraron que el $23,2 \%$ de los sujetos se autolesionaban y el 13,5\% habían cometido un intento suicida en algún momento de la vida, con una asociación significativa entre la autoagresión y trastornos relacionados con el uso de sustancias psicoactivas (20).

En nuestro estudio la proporción de conductas suicidas en adolescentes con EAT- $26>20$ que participaron en la segunda fase del estudio fue significativamente mayor en el grupo con diagnóstico clínico de TCA respecto al grupo con otros diagnósticos psiquiátricos y a los que no tuvieron diagnóstico psiquiátrico por entrevista clínica. Aunque el número de sujetos en esta fase fue limitado, tal hallazgo es consistente con investigaciones de otros países, que encuentran una alta repetición de comportamientos suicidas en población con TCA $(36,37)$. Se ha evidenciado, incluso, una relación entre insatisfacción corporal, patrones inadecuados de alimentación e ideación suicida, lo cual ha llevado a proponer que estos factores se incluyan en los modelos de riesgo suicida en adolescentes (19).

Algunos investigadores sugieren que los comportamientos suicidas en TCA podrían estar mediados por variables sociodemográficas y clínicas, como impulsividad, eventos traumáticos y sintomas depresivos, entre otros. La influencia de estas variables no fue analizada en nuestro estudio; no obstante, los resultados 
destacan la importancia de evaluar el riesgo suicida en jóvenes con TCA; incluso, en centros de atención primaria.

Investigaciones sobre la influencia de actitudes alimentarias de miembros de la familia en la aparición de sintomas a edades tempranas han mostrado que la insatisfacción corporal y la internalización del ideal de delgadez maternos, así como el índice de masa corporal en ambos padres, predicen la aparición de síntomas de patología alimentaria en la infancia precoz. También se ha hallado asociación entre comentarios críticos hacia la figura y el peso por parte de los padres y bulimia en los hijos (12).

Los antecedentes familiares de TCA, la obesidad, las críticas y las bromas acerca del peso y la figura corporal, evaluados por autoinforme en este estudio, concuerdan con los descritos en la literatura internacional y nacional $(8,12)$. Los conocimientos inadecuados sobre alimentación, las actitudes negativas hacia la delgadez y su relación con el éxito, y la aprobación externa fueron también más habituales en jóvenes con diagnóstico probable de TCA en el presente estudio. Algunos de estos factores son potencialmente modificables y respaldan la pertinencia de programas de prevención dirigidos a los jóvenes y sus familias, aunque la evidencia sobre su efectividad aún no sea contundente (3).

La participación en la entrevista psiquiátrica estructurada fue plan- teada a los sujetos de esta investigación de manera voluntaria. No obstante, una limitación importante es que sólo el $38 \%$ de los sujetos detectados como casos probables de TCA aceptaron la entrevista; por eso, los resultados no pueden generalizarse a toda la población afectada por esta patología. Una explicación posible de tal rechazo sería la alta resistencia de los jóvenes y sus familias al contacto con la psiquiatría. Sin embargo, es probable que el $62 \%$ restante de sujetos con EAT $26<20$ estuvieran más o menos "enfermos", o en mayor o menor riesgo de patología alimentaria. Debido a que en esta investigación no se exploraron los motivos de la renuencia a la entrevista, no es posible hacer afirmaciones en ningún sentido.

Las pruebas de tamizaje de TCA en adolescentes han mostrado una sobrevaloración del riesgo, debido a la tendencia de los adolescentes a exagerar las respuestas. Por eso, los resultados de la primera fase de este estudio, específicamente los de factores de riesgo individual y familiar de TCA, deben interpretarse con cautela.

El estudio representa un gran esfuerzo pertinente y útil para caracterizar el problema de conductas de riesgo de trastornos alimentarios en población de jóvenes escolarizados de Bogotá y Cundinamarca. Debe también alertar a la comunidad médica y educativa acerca de la necesidad de implementar de manera permanente programas de prevención. 


\section{Referencias}

1. Gil M, Canalda J, Villanueva C, Rodríguez M. Atención primaria y trastornos de la alimentación: nuestra actitud frente a ellos (I). Aten Primaria. 2003;31(3):178-80.

2. Austin SB. Prevention research in eating disorders: theory and new directions. Psychol Med. 2000;30(6):1249-62.

3. Pratt BM, Woolfenden SR. Interventions for preventing eating disorders in childrens and adolescents. Cochrane Database Syst Rev. 2002;(2):CD002891.

4. Strober M, Freeman R, Lampert C, Diamond J, Kaye W. Controlled family study of anorexia nervosa and bulimia nervosa: evidence of shared liability and transmission of partial syndromes. Am J Psychiatry. 2000;157(3):393-401.

5. Stein D, Meged S, Bar-Hanin T, Blank S, Elizur A, Weizman A. Partial eating disorders in a community sample of female adolescents. J Am Acad Child Adolesc Psychiatry. 1997;36 (8):1116-23.

6. Lewinsohn PM, Striegel-Moore RH, Seeley JR. Epidemiology and natural course of eating disorders in young women from adolescence to young adulthood. J Am Acad Child Adolesc Psychiatry. 2000;39(10):1284-92.

7. Yepes M, Moreno S. Ramírez L. Corporalidad y desarrollo subjetivo en la prevención de la anorexia y la bulimia en las adolescentes escolarizadas de la ciudad de Medellín. Fase de Comprensión. Revista de la Salud por los Niños de las Américas Saludarte. 2002;6(3):23-397.

8. Ángel L, Vásquez R, Chavarro K, Martínez L, García J. Prevalencia de trastornos del comportamiento alimentario en estudiantes de la Universidad Nacional de Colombia entre julio de 1994 y diciembre de 1995. Acta Med Colomb. 1997;22(3):111-9.
9. Rueda G, Díaz L, Ortiz DP, Pinzón C, Rodríguez J, Cadena L. Validación del cuestionario SCOFF para el cribado de los trastornos del comportamiento alimentario en adolescentes escolarizadas. Aten Primaria. 2005;35(2):89-94.

10. Rueda G, Díaz L, Campo A, Barros J, Ávila $\mathrm{G}$, Oróstegui L, et al. Validación de la Encuesta Scoff para tamizaje de trastornos de la conducta alimentaria en mujeres universitarias. Biomédica. 2005;25(2):196-202.

11. Wentz E, Gillberg C, Gillberg IC, Rastam M. Ten-year follow-up of adolescent-onset anorexia nervosa: psychiatric disorders and overall functioning scales. J Child Psychol Psychiatry. 2001;42(5):613-22.

12. Jacobi C, Agras W, Hammer L. Predicting children's reported eating disturbances at 8 years of age. $J$ Am Acad Child Adolesc Psychiatry. 2001;43(3):364-72.

13. Stice E. Risk and maintenance factors for eating pathology: a meta-analytic review. Psychol Bull. 2002;128(5):825-48.

14. Stice E, Presnell K, Bearman S. Relation of early menarche to depression, eating disorders, substance abuse, and comorbid psychopathology among adolescent girls. Dev Psychol. 2001;37(5):608-19.

15. Fedorowicz V, Falissard B, Foulon C, Dardennes R, Divac S, Guelfi J, et al. Factors associated with suicidal behaviors in a large french sample of inpatients with eating disorders. Int $\mathrm{J}$ Eat Disord. 2007;40(7):589-95.

16. Crow S, Eisenberg ME, Story $M$, Neumark-Sztainer D. Suicidal behavior in adolescents: relationship to weight status, weight control behaviors, and body dissatisfaction. Int J Eat Disord. 2008;41(1):82-7.

17. Ángel L, Vásquez R. Trastornos del comportamiento alimentario: caracte- 
rísticas clínicas y evolución. Acta Méd Colomb. 1995;20(1):14-21.

18. Vásquez R, Angel L, Moreno N, García $\mathrm{J}$, Calvo M. Estudio descriptivo de un grupo de pacientes con anorexia nerviosa. Actual Pediatr. 1998;8(1):7-14.

19. Corcos M, Taïeb O, Benoit S, Paterniti $S$, Jeammet P, Flament M. Suicide attempts in women with bulimia nervosa: Frequency and characteristics. Acta Psychiatr Scand. 2002;106(5):381-6.

20. Rodríguez M. Uso de sustancias, impulsividad y trauma en pacientes con trastornos del comportamiento alimentario: una tríada de riesgo para comorbilidades complejas que afectan el pronóstico. Rev Colomb Psiquiatr. 2009;38(3):420-32.

21. Molano J, Piñeros S, López de Mesa C. Dispepsia y reflujo gastroesofágico en adolescentes escolarizados. Rev Col Gastroenterol. 2008;23(1):46-56.

22. Molano J, Piñeros S, López de Mesa C. Factores emocionales e impacto sobre la escolaridad y actividad social en adolescentes con dispepsia y reflujo gastroesofágico. Rev Col Gastroenterol. 2009;24(4):364-72.

23. Garfinkel $P$, Newman A. The eating attitudes test: twenty years later. Eat Weight Disord. 2000;6(1):1-24.

24. Garner DM, Garfinkel PE. The Eating Attitudes Test: an index of the symptoms of anorexia nervosa. Psychol Med. 1979;9(2):273-9.

25. Castrillón MD, Luna I, Aguirre D. Validación del Abbreviated Eating Attitudes (Escala abreviada y modificada de las actitudes alimentarias) EAT-26- M para la población colombiana. En: Ferrer BA, Gómez MY (editores). Evaluación e intervención en niños y adolescentes: investigación y conceptualización. Medellín: La Carreta; 2007. p. 93-116.
26. Zung WW. A self-report depression scale. Arch Gen Psychiatry. 1965;12:63-70.

27. Torres Y, Murelle L. Estudio Nacional sobre Alcoholismo y Consumo de Sustancias Psicoactivas-Colombia 1987. Medellín: Universidad de Antioquia; 1988.

28. Torres Y, Posada J. Estudio Nacional de Salud Mental y Consumo de Sustancias Psicoactivas. Bogotá: Ministerio de Salud; 1993.

29. King MB. Eating disorders in a general practice population. Prevalence, characteristics and follow up at 12 to 18 months. Psychol Med Monogr Suppl. 1989;14:1-34.

30. King M. The natural history of eating pathology in attenders to primary medical care. Int J Eat Disord. 1991;10(4): 379-87.

31. Latzer Y, Vander, Gilat I. Socio-demographic characteristics of eating disorder patients in an outpatient clinic: a descriptive epidemiological study. Eur Eat Disord Rev. 2008;16(2):139-46.

32. Keel PK, Klump KL, Miller KB, McGue $M$, lacono WG. Shared transmission of eating disorders and anxiety disorders. Int J Eat Disord. 2005;38(2):99-105.

33. Silberg JL, Bulik CM. The developmental association between eating disorders symptoms and symptoms of depression and anxiety in juvenile twin girls. J Child Psychol Psychiatry. 2005;46(12):1317-26.

34. Raney TJ, Thornton L, Berrettini W, Brandt $\mathrm{H}$, Crawford S, Fichter $\mathrm{M}$, et al. Influence of overanxious disorder of childhood on the expression of anorexia nervosa. Int $\mathrm{J}$ Eat Disord. 2008;41(4):326-32.

35. Fletcher GA, Kupshik S, Uprichard S, Shah S, Nash A. Eating disorders and 
concurrent psychopathology: a reconceptualisation of clinical need through Rasch Analysis. Eur Eat Disord Rev. 2008;16(3):191-8 .

36. Miotto P, de Coppi M, Frezza M, Pretty A. Eating disorders and suicide risk factors in adolescents: an Italian community-based study. J Ner Ment Dis. 2003;191(7):437-43.
37. Crow S, Eisenberg ME, Story $M$, Neumark Sztainer D. Are body dissatisfaction, eating disturbance, and body mass index predictors of suicidal behavior in adolescents? a longitudinal study. J Consult Clin Psychol. 2008;76(5):887-92.

Conflicto de interés: las autoras manifiestan que no tienen ningún conflicto de interés en este artículo.

Recibido para evaluación: 2 de febrero del 2010 Aceptado para publicación: 29 de abril del 2010

Correspondencia Sandra Piñeros Ortiz Facultad de Medicina Universidad de La Sabana Campus Universitario del Puente del Común Kilómetro 7, autopista Norte Chia, Cundinamarca, Colombia sandrapinor@hotmail.com 